\title{
Status of a minimal composite Higgs theory
}

\author{
Zoltan Fodor \\ University of Wuppertal, Department of Physics, Wuppertal D-42097, Germany \\ Juelich Supercomputing Center, Forschungszentrum Juelich, Juelich D-52425, Germany \\ Eotvos University, Pazmany Peter setany 1, 1117 Budapest, Hungary \\ fodorabodri.elte. hu
}

Kieran Holland

University of the Pacific, 3601 Pacific Ave, Stockton CA 95211, USA

Albert Einstein Center for Fundamental Physics, Bern University, Bern, Switzerland

khollandepacific.edu

Julius Kuti ${ }^{*}$

University of California, San Diego, 9500 Gilman Drive, La Jolla, CA 92093, USA

jkuti@ucsd.edu

\section{Santanu Mondal ${ }^{*}$}

Eotvos University, Pazmany Peter setany 1, 1117 Budapest, Hungary

MTA-ELTE Lendulet Lattice Gauge Theory Research Group, 1117 Budapest, Hungary

santanu@bodri.elte.hu

\section{Daniel Nogradi}

Eotvos University, Pazmany Peter setany 1, 1117 Budapest, Hungary

MTA-ELTE Lendulet Lattice Gauge Theory Research Group, 1117 Budapest, Hungary

nogradi@bodri.elte.hu

\section{Chik Him Wong*}

University of Wuppertal, Department of Physics, Wuppertal D-42097, Germany

cwong@uni-wuppertal.de

We analyze three sets of gauge ensembles in our extended physics program of a particularly important BSM gauge theory with a fermion doublet in the two-index symmetric (sextet) representation of the SU(3) BSM color gauge group. Our investigations include chiral symmetry breaking $(\chi \mathrm{SB})$ in the p-regime and $\varepsilon$-regime, the mass of the composite $0^{++}$scalar, resonance spectroscopy, new physics from gauge anomaly constraints, and the role of stable sextet BSM baryons with Electroweak interactions in dark matter searches. Important new goals include studies of the $0^{++}$scalar entangled with Goldstone dynamics in the p-regime and the $\varepsilon$-regime, the resonance spectrum with particular attention to emerging LHC signals, like recent hints for diphoton excess at $750 \mathrm{GeV}$ or diboson anomalies in the $2 \mathrm{TeV}$ range. All results reported here are preliminary before journal publication including some post-conference material for the discussion.

The 33rd International Symposium on Lattice Field Theory,

14 - 18 July 2015

Kobe International Conference Center, Kobe, Japan

\footnotetext{
* Speaker.
} 


\section{Introduction}

An important strongly coupled near-conformal gauge theory built on the minimally required $\mathrm{SU}(2)$ bsm-flavor doublet of two massless fermions, with a confining gauge force at the TeV scale in the sextet representation of the new SU(3) BSM color gauge group is an intriguing possibility for the minimal realization of the composite Higgs mechanism. Early discussions of the model as a BSM candidate were initiated in systematic explorations of higher fermion representations of color gauge groups [1-3] for extensions of the original Higgsless Technicolor paradigm [4,5]. In fact, the first appearance of the particular two-index symmetric SU(3) fermion representation can be traced even further back to Quantum Chromodynamics (QCD) where a doublet of sextet quarks was proposed as a mechanism for Electroweak symmetry breaking (EWSB) without an elementary Higgs field [6]. This idea had to be replaced by a new gauge force at the TeV scale, orders of magnitude stronger than in QCD, to facilitate the dynamics of EWSB just below the lower edge of the conformal window in the new BSM paradigm [1-3]. It should be noted that throughout its early history the important near-conformal behavior of the model was not known and definitive results had to wait for recent non-perturbative investigations with lattice gauge theory methods as used in our work.

Near-conformal BSM theories raise the possibility of a light composite scalar, perhaps a Higgs impostor, to emerge from new strong dynamics, far separated from the associated composite resonance spectrum in the few $\mathrm{TeV}$ mass range with interesting and testable predictions for the Large Hadron Collider (LHC). This scenario is very different from what was expected from QCD when scaled up to the Electroweak scale, as illustrated by the failure of the Higgsless Technicolor paradigm. Given the discovery of the $125 \mathrm{GeV}$ Higgs particle at the LHC, any realistic BSM theory must contain a Higgs-like state, perhaps with some hidden composite structure.

Based on our $a b$ initio non-perturbative lattice calculations we find accumulating evidence for near-conformal behavior in the sextet theory with the emergent low mass $0^{++}$scalar state far separated from the composite resonance spectrum of bosonic and baryonic excitations in the 2-3 TeV energy range [7-10]. The identification of the light scalar state is numerically challenging since it requires the evaluation of disconnected fermion loop contributions to correlators with vacuum quantum numbers in the range of light fermion masses we explore. The evidence to date is very promising that the $0^{++}$scalar is light in the chiral limit and that the model at this stage remains an important BSM candidate. This report presents new results and outlines the need for important further work needed for definitive results.

In Section 2 for the first time we describe the anomaly-free Electroweak embedding of the strongly coupled sextet gauge theory in a more comprehensive way than before and present the need for more extensive baryon analysis of our gauge ensembles with relevance for dark matter searches and model viability. In Section 3 new results are reported and analysis tools are proposed for mass-deformed chiral perturbation theory and its chiral limit using p-regime and $\varepsilon$-regime technologies including the improved chiral properties of mixed actions in the valence sector. This is critically important for accurate BSM scale setting from the chiral limit of the Goldstone decay constant $\mathrm{F}_{\pi}$ and the chiral condensate. In Section 4 the status of the light $0^{++}$scalar and the resonance spectrum is discussed with new plans briefly outlined. Section 5 describes efforts to understand physics at several scales in nearly conformal gauge theories from the asymptotically free 
UV regime with an almost conformal crossover to the infrared with spontaneous chiral symmetry breaking. Flavor dependence of the running coupling and beta function in the sextet and fundamental fermion representations illustrates this problem. In Section 6 we provide a brief summary of the computational aspects of our work describing the staggered fermion action we use in the simulations with well known taste breaking effects on the exact chiral symmetries of the continuum theory. All figures are attached at the end of the report without interrupting the flow of the narrative.

\section{Electroweak multiplet structure, gauge anomalies, and baryons}

As in the minimal scheme of Susskind [4] and Weinberg [5], the gauge group of the theory is $\mathrm{SU}(3)_{\mathrm{bsm}} \otimes \mathrm{SU}(3)_{\mathrm{c}} \otimes \mathrm{SU}(2)_{\mathrm{w}} \otimes \mathrm{U}(1)_{\mathrm{Y}}$ where $\mathrm{SU}(3)_{\mathrm{c}}$ designates the QCD color gauge group and $\mathrm{SU}(3)_{\mathrm{bsm}}$ represents the BSM color gauge group of the new strong gauge force. In addition to quarks and leptons of the Standard Model, we include one SU(2) bsm-flavor doublet (u,d) of fermions which are $\mathrm{SU}(3)_{\mathrm{c}}$ singlets and transform in the six-dimensional sextet representation of BSM color, distinct from the fundamental color representation of fermions in the original Technicolor scheme [4,5]. The formal designation $(\mathrm{u}, \mathrm{d})$ for the bsm-flavor doublet of sextet fermions uses a similar notation to the two light quarks of QCD but describes completely different physics. The massless sextet fermions form two chiral doublets $(u, d)_{L}$ and $(u, d)_{R}$ under the global symmetry group $\mathrm{SU}(2)_{\mathrm{L}} \otimes \mathrm{SU}(2)_{\mathrm{R}} \otimes \mathrm{U}(1)_{\mathrm{B}}$. Baryon number is conserved for quarks of the Standard Model separate from baryon number conservation for sextet fermions which carry $1 / 3$ of BSM baryon charge associated with the BSM sector of the global $\mathrm{U}(1)_{\mathrm{B}}$ symmetry group.

\subsection{Electroweak multiplet structure}

It is straightforward to define consistent multiplets for the sextet fermion flavor doublet under the $\mathrm{SU}(2)_{\mathrm{w}} \otimes \mathrm{U}(1)_{\mathrm{Y}}$ Electroweak gauge group with hypercharge assignments for left- and righthanded fermions transforming under the $\mathrm{SU}(2)_{\mathrm{w}}$ weak isospin group. The two fermion flavors $\mathrm{u}^{\mathrm{ab}}$ and $\mathrm{d}^{\mathrm{ab}}$ of the strongly coupled sector carry six colors in two-index symmetric tensor notation, $\mathrm{a}, \mathrm{b}=1,2,3$, associated with the gauge force of the $\mathrm{SU}(3)_{\mathrm{bsm}}$ group. This is equivalent to a sixdimensional vector notation in the sextet representation. The fermions transform as left-handed weak isospin doublets and right-handed weak isospin singlets for each color,

$$
\psi_{\mathrm{L}}^{\mathrm{ab}}=\left(\begin{array}{c}
\mathrm{u}_{\mathrm{L}}^{\mathrm{ab}} \\
\mathrm{d}_{\mathrm{L}}^{\mathrm{ab}}
\end{array}\right), \quad \psi_{\mathrm{R}}^{\mathrm{ab}}=\left(\mathrm{u}_{\mathrm{R}}^{\mathrm{ab}}, \mathrm{d}_{\mathrm{R}}^{\mathrm{ab}}\right) .
$$

With this choice of representations, the normalization for the hypercharge $\mathrm{Y}$ of the $\mathrm{U}(1)_{\mathrm{Y}}$ gauge group is defined by the relation $\mathrm{Y}=2\left(\mathrm{Q}-\mathrm{T}_{3}\right)$, with $\mathrm{T}_{3}$ designating the third component of weak isospin.

Once Electroweak gauge interactions are turned on, the chiral symmetry breaking pattern $\mathrm{SU}(2)_{\mathrm{L}} \otimes \mathrm{SU}(2)_{\mathrm{R}} \rightarrow \mathrm{SU}(2)_{\mathrm{V}}$ of strong dynamics breaks Electroweak symmetry in the expected pattern, $\mathrm{SU}(2)_{\mathrm{w}} \times \mathrm{U}(1)_{\mathrm{Y}} \rightarrow \mathrm{U}(1)_{\mathrm{em}}$, and with the simultaneous dynamical realization of the composite Higgs mechanism. It is important to note that the dynamical Higgs mechanism is facilitated through the electroweak gauge couplings of the sextet fermions and does not depend on the hypercharge assignments of the multiplets [4]. Recently, we presented a detailed analysis on anomaly 
constraints of hypercharge assignments [11]. In this report we summarize what is relevant for the sextet baryon analysis of our existing gauge ensembles with dark matter and model viability implications [11].

\subsection{Anomaly conditions}

Anomaly constraints have a long history in Technicolor motivated BSM model building with representative examples in $[1,12-15]$. The first condition for model construction with left-handed doublets is the global Witten anomaly constraint which requires an even number of left-handed $\mathrm{SU}(2)$ multiplets to avoid inconsistency in the theory from a vanishing fermion determinant of the partition function [16]. In addition, gauge anomaly constraints also have to be satisfied [17]. With vector current $\mathrm{V}_{\mu}^{\mathrm{i}}(\mathrm{x})=\bar{\psi} \mathrm{T}^{\mathrm{i}} \gamma_{\mu} \psi(\mathrm{x})$ and axial current $\mathrm{A}_{\mu}^{\mathrm{i}}(\mathrm{x})=\bar{\psi} \mathrm{T}^{\mathrm{i}} \gamma_{\mu} \gamma_{5} \psi(\mathrm{x})$ constructed from fermion fields and internal symmetry matrices $\mathrm{T}^{\mathrm{i}}$ in some group representation $\mathrm{R}$ for fermions, the anomaly in the axial vector Ward identity is proportional to $\operatorname{tr}\left(\left\{\mathrm{T}^{\mathrm{i}}(\mathrm{R}), \mathrm{T}^{\mathrm{j}}(\mathrm{R})\right\} \mathrm{T}^{\mathrm{k}}(\mathrm{R})\right)$ and must vanish. In the sextet theory fermions are either left-handed doublets or right-handed singlets under the $\mathrm{SU}(2)_{\mathrm{w}}$ gauge group. The matrices $\mathrm{T}^{\mathrm{i}}$ will be either the $\tau^{\mathrm{i}}$ Pauli matrices or the diagonal $\mathrm{U}(1)$ hypercharge Y. Since the $\mathrm{SU}(2)$ group is anomaly free, $\operatorname{tr}\left(\left\{\tau^{\mathrm{i}}, \tau^{\mathrm{j}}\right\} \tau^{\mathrm{k}}\right)=0$, we only need to consider anomalies where at least one $\mathrm{T}^{\mathrm{i}}$ is the hypercharge $\mathrm{Y}$. The non-trivial constraints come from two conditions on hypercharge traces,

$$
\operatorname{tr}(\mathrm{Y})=0, \quad \operatorname{tr}\left(\mathrm{Y}^{3}\right) \propto \operatorname{tr}\left(\mathrm{Q}^{2} \mathrm{~T}_{3}-\mathrm{QT}_{3}^{2}\right)=0
$$

where $\mathrm{Y}=2\left(\mathrm{Q}-\mathrm{T}_{3}\right)$ with electric charge $\mathrm{Q}$, and $\mathrm{T}_{3}$ as the third component of weak isospin. There are two simple solutions for BSM model building with sextet fermions to satisfy the Witten anomaly condition and gauge anomaly constraints on $\operatorname{tr}(\mathrm{Y})$ and $\operatorname{tr}\left(\mathrm{Y}^{3}\right)$ in Eq. (2.2). The first solution with the choice $\mathrm{Y}\left(\mathrm{f}_{\mathrm{L}}\right)=0$ for doublets of left-handed sextet fermions $\left(\mathrm{f}_{\mathrm{L}}\right)$ leads to halfinteger electric charges for composite baryons. The second solution with the choice $\mathrm{Y}\left(\mathrm{f}_{\mathrm{L}}\right)=1 / 3$ for doublets of left-handed sextet fermions leads to integer electric charges for composite baryons. The hypercharges of right-handed singlets are automatically set from consistent electric charge assignments in both cases. The two choices have very different implications for sextet baryons.

\subsection{Sextet baryons and their Early Universe}

In the sextet BSM theory we do not have direct observations of new heavy baryons to set unique hypercharge assignments for left-handed doublets and right-handed singlets of sextet fermions from two alternate solutions to the anomaly conditions. Viability of the choices $Y\left(f_{L}\right)=0$, or $\mathrm{Y}\left(\mathrm{f}_{\mathrm{L}}\right)=1 / 3$, is affected by the different electric charge assignments they imply. With heavy sextet baryon masses in the $3 \mathrm{TeV}$ range, as determined from our recent lattice simulations [11], the seemingly minimal solution with $\mathrm{Y}=0$ for left-handed doublets would lead to intriguing predictions of baryon states with half-integer electric charges for future accelerator searches and relics with fractional electric charges from the early Universe with observable consequences. Problems with half-integer electric charges, from the choice $\mathrm{Y}\left(\mathrm{f}_{\mathrm{L}}\right)=0$ in our case, were anticipated earlier from strong observational limits on stable fractional charges in the early Universe and their terrestrial relics $[18,19]$. The non-controversial $\mathrm{Y}\left(\mathrm{f}_{\mathrm{L}}\right)=1 / 3$ anomaly solution for the sextet model has new dark matter implications [11] which require new lattice calculations proposed here. 


\subsection{Ongoing lattice work on sextet baryons and future plans}

The lightest baryons in the strongly coupled sextet gauge sector are expected to form isospin flavor doublets (uud, udd), similar to the pattern in QCD. As we noted earlier, baryons in the sextet model should carry integer multiples of electric charges if $\mathrm{Y}\left(\mathrm{f}_{\mathrm{L}}\right) \neq 0$ to avoid problems with the relics of the early Universe. This leads to the simplest choice $\mathrm{Y}\left(\mathrm{f}_{\mathrm{L}}\right)=1 / 3$ with gauge anomalies to be compensated. A new pair of left-handed lepton doublets emerged from this choice as the simplest manifestation of the anomalies and the Electroweak extension of the strongly coupled sextet gauge sector [11].

Neutron-like udd sextet model baryons $\left(\mathrm{n}_{6}\right)$ will carry no electric charge and proton-like uud sextet model baryons $\left(p_{6}\right)$ have one unit of positive electric charge from the choice $Y\left(f_{L}\right)=1 / 3$. The two baryon masses are split by electromagnetic interactions. The ordering of the two baryon masses in the chiral limit of massless sextet fermions will require non-perturbative $a b$ initio lattice calculations of the electromagnetic mass shifts to confirm intuitive expectations that the neutronlike $\mathrm{n}_{6}$ baryon has lower mass than the proton-like $\mathrm{p}_{6}$ baryon. In QCD this pattern was confirmed by recent lattice calculations [20]. We expect the same ordering in the sextet model so that the protonlike $\mathrm{p}_{6}$ baryon will decay very fast, $\mathrm{p}_{6} \rightarrow \mathrm{n}_{6}+\ldots$, with a lifetime $\tau \ll 1$ second. It is unlikely for rapidly decaying $\mathrm{p}_{6}$ baryons to leave any relic footprints from dark nucleosynthesis before they decay.

With BSM baryon number conservation the neutral $\mathrm{n}_{6}$ baryon is stable and observational limits on its direct detection from experiments like XENON100 [21] and LUX2013 [22] have to be estimated. In charge symmetric thermal evolution sextet model baryons are produced with relic number density ratio $\mathrm{n}_{\mathrm{B}_{6}} / \mathrm{n}_{\mathrm{B}} \approx 3 \cdot 10^{-7}$. For $3 \mathrm{TeV}$ sextet model baryon masses we can estimate the detectable dark matter ratio of respective mass densities $\rho_{\mathrm{B}_{6}}$ and $\rho_{\mathrm{B}}$ as $\rho_{\mathrm{B}_{6}} / \rho_{\mathrm{B}} \approx 10^{-4}$, about $5 \cdot 10^{4}$ times less than the full amount of unaccounted dark mass, $\rho_{\text {dark }} \approx 5 \cdot \rho_{\mathrm{B}}$. We will use this mass density estimate to guide observational limits on relic sextet model baryons emerging from charge symmetric thermal evolution where tests of dark baryon detection come from elastic collisions with nuclei in dark matter detectors [11]. The neutral and stable $\mathrm{n}_{6}$ baryon can interact several different ways with heavy nuclei in direct detection experiments including (a) magnetic dipole interaction, (b) Z-boson exchange, (c) Higgs boson exchange, and (d) electric polarizability. It turns out that cross sections from (a) and (b) can be parametrized and estimated even without lattice simulations. Cross sections from (c) and (d) require lattice calculations using our existing gauge ensembles and capacity computing from new allocation we request for gpu capacity computing. Based on these estimates we expect to show that the sextet BSM model is consistent with observational limits and stable baryons will contribute a small fraction to the missing dark matter content. New physics implied by gauge anomaly constraints, like new lepton generations with neutrinos [11], can also contribute to the relic abundance of dark matter. These are important and interesting issue for future investigations.

\section{Mass-deformed chiral perturbation theory and the chiral condensate}

One of the most important goals of lattice BSM models is to accurately set the Electroweak scale as a function of the lattice spacing. This allows control on the continuum limit when the cutoff 
is removed and phenomenologically relevant BSM predictions are made. The chiral $\mathrm{SU}(2)_{\mathrm{L}} \times \mathrm{SU}(2)_{\mathrm{R}}$ symmetry of the model is dynamically broken to the diagonal vector symmetry $\mathrm{SU}(2)_{\mathrm{V}}$ and three associated Goldstone pions facilitate the minimal realization of the Higgs mechanism after the Electroweak interactions are turned on. The Electroweak scale in finite lattice spacing units is set from the decay constant $F_{\pi}$ of the Goldstone pion in the chiral limit with $F=250 \mathrm{GeV}$ in continuum physics notation. It can be identified as the fundamental scale of the theory related to the chiral (Higgs) condensate through the GMOR relation.

\subsection{Taste breaking cutoff effects in the staggered pion spectrum}

Since the determination of the Goldstone decay constant $\mathrm{F}$ in the chiral limit is critically important for the location of the light scalar mass and the well-separated resonance spectrum in the 2-3 TeV range, we carefully monitor taste breaking effects in the pion spectrum with the goal of removing cutoff effects from physics predictions. This also serves as guidance for our choice of lattice spacings for new configuration generation.

To illustrate cutoff dependent taste breaking effects, spectra of mass-deformed non-Goldstone pion states are shown in Figure 1 from our newest data with the definition of the relevant correlators and quantum numbers given in $[7,30]$. In the fermion mass range of our data set the taste breaking pattern is different from QCD where the residual $\Delta$ mass shifts of the non-Goldstome pions are equispaced in the chiral limit with approximately degenerate $\mathrm{SO}(4)$ taste multiplets and with parallel slopes for finite fermion mass deformations of Goldstone and non-Goldstone pion states [29]. For example, as part of the equispaced split of degenerate $\mathrm{SO}(4)$ multiplets, the observed approximate split $\Delta_{\mathrm{ij}} \sim 2 \Delta_{\mathrm{sc}}$ of two multiplets in QCD appears to have collapsed in the sextet model. The other distinct difference from QCD is the non-parallel slopes which fan out in Goldstone and nonGoldstone mass deformations of the pion spectrum as shown in Figure 1. While the $\Delta$ additive mass shifts are LO taste breaking effects in the chiral Lagrangian [29,31], the taste breaking slope corrections $\delta$ can plausibly be identified with NLO analytic terms in rooted staggered chiral perturbation theory (rs $\chi \mathrm{PT}$ ) [32]. The corrected mass relation is $\mathrm{M}_{\mathrm{NLO}}^{2}=\mathrm{M}_{\mathrm{LO}}^{2}(1+\delta)$ where $\delta$ depends on the taste quantum number of the pion state. Several relations constrain the $\delta$ taste breaking corrections [32]. The pion spectrum with taste breaking cutoff effects is the input to analyze the fundamental parameters of rs $\chi \mathrm{PT}$ as worked out for the SU(3) group in [31]. Our adaptation to the $\mathrm{SU}(2)$ group of $\mathrm{rs} \chi \mathrm{PT}$ in the sextet model is straightforward.

\subsection{Fundamental parameters from rooted staggered chiral perturbation theory (p-regime)}

For the SU(2) analysis we adapted the procedure from [31]. There are two fundamental parameters $\mathrm{F}$ and $\mathrm{B}$ in the $\mathrm{SU}(2)$ chiral Lagrangian. The fundamental parameter $\mathrm{F}$ of $\chi \mathrm{PT}$, defined as the chiral limit of the pion decay constant $\mathrm{F}_{\pi}$, sets the Electroweak scale and the fundamental parameter B sets the fermion mass deformation of the Goldstone spectrum. With bare fermion mass $\mathrm{m}$, the $\mathrm{RG}$ invariant combination $\mathrm{m} \cdot \mathrm{BF}^{2}$ is related to the chiral condensate via the GMOR relation.

We apply rooted staggered chiral perturbation theory to the mass-deformed pion spectrum and $\mathrm{F}_{\pi}$. The fitting procedure in the p-regime proceeds in several steps. In the first step finite volume correction is applied to the $\mathrm{M}_{\pi}$ and $\mathrm{F}_{\pi}$ data from 1-loop continuum $\chi \mathrm{PT}$. This is sufficient to assure 
that in the next step the fitting procedure is applied to data free from volume dependence. A linear fit is applied to the quadratic masses of the non-Goldstone pion spectrum to determine their mass shifts and slopes. In the final analysis of rooted chiral perturbation theory, non-Goldstone pion states run in the chiral loops including their mass splittings and fan-out slope structure from taste breaking as determined from the linear fits to the non-Goldstone spectrum. We applied this analysis at two values of the gauge coupling where we have extensive ensembles.

For illustration, preliminary results from rs $\chi \mathrm{PT}$ are shown in Figure 2 from fits at gauge coupling $\beta=3.20$ which corresponds to our coarser lattice of the two extended sets of gauge ensembles. The upper left panel shows the linear fits to the quadratic masses of the non-Goldstone pions to determine their mass shifts and slopes as input. The upper right panel shows the rs $\chi$ PT fit to $\mathrm{F}_{\pi}$ as a function of fermion mass deformations away from the chiral limit. The two lower panels show rs $\chi \mathrm{PT}$ fits to $\mathrm{M}_{\pi}$ as a function of fermion mass deformations away from the chiral limit. Fits at the finer lattice spacing $\beta=3.25$ are quite similar in quality but with lower confidence level. The unambiguous determination of the cutoff dependent $\mathrm{F}$ and $\mathrm{B}$ parameters and their continuum limit from rs $\chi \mathrm{PT}$ will require extended analysis. Partial quenching with valence fermions is the first added step to make the ongoing analysis more robust.

Although our results are consistent with chiral symmetry breaking and rs $\chi \mathrm{PT}$, ongoing work will require considerable extensions for definitive results. Important new work, besides partial quenching includes a solution to the entanglement problem of the light scalar with the low pion spectrum in perturbation theory with comparable masses in the rs $\chi \mathrm{PT}$ regime, crossover analysis from the p-regime to the $\varepsilon$-regime and applications of Random Matrix Theory (RMT) in the $\varepsilon$ regime. Coupled chiral dynamics of the low mass scalar $0^{++}$state with the pions requires new analysis based on an extended effective theory. We are using the modified effective field theory of $\chi \mathrm{PT}$ on existing gauge ensembles but new gauge configuration generation is also needed in the crossover to the $\varepsilon$ regime. For independent control on the results for the fundamental parameters $\mathrm{F}$ and $\mathrm{B}$ we developed and apply now mixed actions with improved chiral symmetry without taste breaking in the valence sector of the analysis. Ongoing new efforts in the p-regime and RMT based $\varepsilon$-regime in mixed action setting will resolve important aspects of $\operatorname{rs} \chi \mathrm{PT}$ with better determination of $\mathrm{F}$ and $\mathrm{B}$.

\subsection{Epsilon-regime, RMT, and mixed action in the valence sector}

Safe extrapolation from the entangled regime of the low mass $0^{++}$scalar with pions to the massless fermion limit is enabled by crossover to the $\varepsilon$-regime of $\chi \mathrm{PT}$ at low enough scales $\lambda$ where Goldstone dynamics begins to decouple from the scalar state. This is demanding and requires significant resources. To control taste breaking we cannot go to lattice spacings coarser than the one set by $\beta=3.20$. The uncertainties in the value of $\mathrm{F} \sim 0.018-0.025$ with limitations from rooted chiral perturbation theory at this lattice spacing requires large $\mathrm{V}=56^{3} \times 96$ and $\mathrm{V}=48^{3} \times 96$ lattice volumes to control the $\mathrm{F} \cdot \mathrm{L} \geq 1$ condition which is necessary for convergent expansion in all regimes of $\chi \mathrm{PT}$, including the $\varepsilon$-regime. Even for our largest $\mathrm{V}=56^{3} \times 96$ and $\mathrm{V}=48^{3} \times 96$ lattice volumes control with $\mathrm{F} \cdot \mathrm{L} \sim 1$ is just barely sufficient. For the lowest fermion mass $\mathrm{m}=0.0010$, we have now at these volumes and at this lattice spacing, the scaling variable $\mathrm{m} \Sigma \mathrm{V} \sim 80$ is very large and more appropriate for the p-regime analysis of $\chi \mathrm{PT}$. Reaching the $\varepsilon$-regime requires substantial decrease in the scaling variable $\mathrm{m} \Sigma \mathrm{V}$ targeting $\mathrm{m}=0.0003$ 
which presents considerable algorithmic challenge for accelerated inversion methods and also calls for mixed action innovation. We deploy accelerated inverters in configuration generation to the $\mathrm{m}=0.0010-0.0003$ range and analyze these configurations with mixed valence actions of good chiral properties as described below. Our limited resources this year allowed us to test these methods without comprehensive deployment for phenomenologically relevant results like the $\mathrm{M}_{0^{++}} / \mathrm{F}$ ratio in the continuum limit.

The $\mathrm{m}=0.0010-0.0003$ range is in the crossover from the p-regime to the $\varepsilon$-regime where known methods of $\chi \mathrm{PT}$ are based on partial quenching and mixed action analysis. For reliable testing, we performed $\chi \mathrm{PT}$ analysis in the crossover to the $\varepsilon$-regime with partial quenching and a mixed valence action with improved chiral symmetries. We take the p-regime gauge configurations of the lowest fermion masses on the largest lattice volumes and analyze the fermion condensate and the Dirac spectrum with valence fermion action where the original gauge link variables are replaced with the ones with a fixed number of small stout steps which corresponds to fixed gradient flow time $t$ in lattice spacing units at each gauge coupling. This strategy can be viewed as a mixed action based analysis with very good chiral properties of the fermion valence action.

The newest test results are shown in Figure 3. The valence action is defined with a large number of very small stout steps which corresponds to gradient flow time $t=3$ in cutoff units. We checked the eigenvalues of the Dirac operator which order into nearly degenerate quartets with the smeared gauge links of the gradient flow. The degenerate eigenvalues follow the index theorem count matching the topology of each gauge configuration as measured from the topological charge operator on the gradient flow. The upper left panel shows the RMT distribution of four degenerate quartets showing that the $\varepsilon$-regime is reached with the scaling variable $\lambda \Sigma_{\text {mixed }} \mathrm{V} \sim 10$ where the fermion mass is replaced by the scale of the gradient flow defined valence Dirac spectrum $(\mathrm{m} \rightarrow \lambda)$. The fermion condensate $\Sigma_{\text {mixed }}$, not RG invariant itself, is consistently determined from the gradient flow defined valence Dirac operator. The upper right panel illustrates the perfect degeneracy of the Goldstone pion with one selected non-Goldstone pion (scPion in the plot). We checked that the degeneracy holds for all non-Goldstone pion states. The lower left panel shows the infrared part of the directly calculated Dirac spectral density on the gauge configurations and its Chebyshev expansion based approximation. The lower right panel shows the pion decay constant $\mathrm{F}_{\pi}$ fitted with the mixed action for fixed sea mass as a function of valence masses. Continued future work is needed for definitive results of BSM phenomenology.

\section{The light $0^{++}$scalar and the resonance spectrum}

The most important goals of our lattice Higgs project are to establish the emergence of the light scalar state with $0^{++}$quantum numbers and the resonance spectrum far separated from the light composite scalar.

\subsection{The light scalar state}

The $\mathrm{f}_{0}$ meson (in QCD terminology) has $0^{++}$quantum numbers and acts as the scalar state in the sextet model ( $\sigma$ particle in QCD). Close to the conformal window, the $\mathrm{f}_{0}$ meson of the sextet model is not expected to be similar to its counterpart in QCD. If it turns out to be light, it can replace the elementary Higgs particle and pose as the Higgs impostor. Two types of different 
$0^{++}$operators, the fermionic one and the gluonic one $\left(0^{++}\right.$glueball $)$, are expected to mix in the relevant correlation functions for mass determination. Such mixing was not included in the pilot study [9] but becomes an important goal of our ongoing effort. We will report our new results without including these mixing effects.

A particular flavor-singlet correlator is needed to capture the $0^{++}$scalar state with vacuum quantum numbers. It requires connected and disconnected diagrams of fermion loop propagators on ensemble gauge configurations. The connected diagram corresponds to the non-singlet correlator $\mathrm{C}_{\text {non-singlet }}(\mathrm{t})$. The correlator of the disconnected diagram is $\mathrm{D}(\mathrm{t})$ at time separation t. The $\mathrm{f}_{0}$ correlator $\mathrm{C}_{\text {singlet }}(\mathrm{t})$ is defined as $\mathrm{C}_{\text {singlet }}(\mathrm{t}) \equiv \mathrm{C}_{\text {non-singlet }}(\mathrm{t})+\mathrm{D}(\mathrm{t})$. The transfer matrix has the spectral decomposition of the $\mathrm{C}_{\text {singlet }}(\mathrm{t})$ correlator in terms of the sum of all energy levels $\mathrm{E}_{\mathrm{i}}\left(0^{++}\right), \mathrm{i}=0,1,2, \ldots$ and their parity partners $\mathrm{E}_{\mathrm{j}}\left(0^{-+}\right), \mathrm{j}=0,1,2, \ldots$ but at large time separation $\mathrm{t}$ the lowest states $\mathrm{E}_{0}\left(0^{++}\right)$and $\mathrm{E}_{0}\left(0^{-+}\right)$dominate. They correspond to $\mathrm{m}_{\mathrm{f}_{0}}$ and $\mathrm{m}_{\eta_{\mathrm{sc}}}$. The relevant non-singlet staggered correlator can be fitted well with non-oscillating $a_{0}$ contribution and oscillating $\pi_{\mathrm{sc}}$ contribution, with the non-Goldstone pion $\pi_{\mathrm{sc}}$ discussed in Section 3. One of the most important new developments in our analysis is to use correlators which project out non-zero momentum states of the scalar. This projection eliminates the vacuum contribution in the disconnected part and improves the mass extraction procedure.

We estimate the connected and disconnected diagrams with stochastic source vectors of fermion propagators. To evaluate the disconnected diagram, we need to calculate closed loops of quark propagators. We introduce $Z_{2}$ noise sources on the lattice where each source is defined on individual time-slice $t_{0}$ for color $a$. The scheme can be viewed as a "dilution" scheme which is fully diluted in time and color and even/odd diluted in space. Results from the original pilot study [9] on $32^{3} \times 64$ lattice volumes at $\beta=3.20$ could only extend down to the lowest fermion mass at $\mathrm{m}=0.003$. From our new analysis some representative examples of $0^{++}$effective mass fits are shown in Figure 4 probing the light scalar closer to the chiral limit than before at fermion mass $\mathrm{m}=0.0015$. The upper left panel is at $\beta=3.20$ with $48^{3} \times 96$ lattice volume and the upper right panel is at $\beta=3.20$ with $56^{3} \times 96$ lattice volume to check against finite volume dependence in this low fermion mass range. The two lower panels of the plot show results at $\beta=3.25$.

Although our original estimate $\mathrm{M}_{0^{++}} / \mathrm{F} \sim 1-3$ for the chiral limit remains consistent with the ongoing new analysis, important further work is needed on the light $\mathrm{f}_{0}$ scalar with $0^{++}$quantum numbers. We want better control on the slowly changing topology of the RHMC algorithm and the related dependence of the extracted masses on the topological quantum numbers of the gauge configurations. We are also in the process of a closely related study of the $\eta^{\prime}$ problem which is particularly interesting and important in the staggered fermion formulation. Fermion mass deformations of the low-lying $\mathrm{f}_{0}$ state and the Goldstone pion are expected to be entangled which requires extended $\chi \mathrm{PT}$ analysis. Our ongoing work will have to address these issues.

\subsection{The emerging resonance spectroscopy}

It is important to investigate the chiral limit of composite hadron states separated from the Goldstones and the light scalar by finite mass gaps. The baryon mass gap in the chiral limit, for example, provides further evidence for $\chi \mathrm{SB}$ with preliminary results reported earlier [24]. Resonance masses of parity partners provide important additional information with split parity masses 
in the chiral limit. This is particularly important for consistency with $\chi \mathrm{SB}$ and for a first estimate of the S parameter when probing the model via Electroweak precision tests [37].

A remarkable resonance spectrum is emerging in our new analysis which is sketched in Figure 5 for illustration only. The scale is set by $\mathrm{F}$ in $\mathrm{TeV}$ units at both lattice spacings with caveats from discussions in Section 3 of the report. Any conclusion about $\chi \mathrm{SB}$ or conformal behavior from eyeballed inspection of the data would be inappropriate and misleading. Although with more work needed for confirmation, the sextet model appears to be close to the conformal window and due to $\chi \mathrm{SB}$ exhibits the right Goldstone spectrum for the minimal realization of the composite Higgs mechanism with a light scalar separated from the associated resonance spectrum in the 2-3 TeV region. Chiral symmetry breaking and a very small beta function are not sufficient to guarantee a light dilaton-like state as the natural interpretation for the emergence of the light scalar. Consistent with our observations, a light Higgs-like scalar is still expected to emerge near the conformal window as a composite state with $0^{++}$quantum numbers, but not necessarily with a dilaton interpretation. This scalar state has to be light but is not required to match exactly the observed $125 \mathrm{GeV}$ mass. The light scalar from composite strong dynamics gets lighter from electroweak loop corrections, dominated by the large negative mass shift from the top quark loop [38-40].

\section{The scale dependent renormalized coupling and beta function}

An important and independent consistency condition of the model would be provided by matching the scale dependent renormalized coupling of the perturbative regime to the scale dependent coupling of the non-perturbative phase associated with $\chi \mathrm{SB}$. We proposed a gauge coupling earlier $g(\mu=1 / L)$, running with the scale set by the finite volume [41] and defined on the gradient flow of the gauge field [42]. Since the gradient flow at flow time $t$ probes the gauge field at the scale $\sqrt{8 t}$, the running coupling can be defined as a function of $L$ in finite volume $V=L^{4}$ while holding $c=(8 t)^{1 / 2} / L$ fixed with $\alpha_{\mathrm{c}}(\mathrm{L})=4 \pi\left\langle\mathrm{t}^{2} \mathrm{E}(\mathrm{t})\right\rangle /[3(1+\delta(\mathrm{c})]$ with details of the definition given in [41]. This volume dependent coupling is particularly suitable to study the perturbative regime and departures from it. The measured renormalized couplings are very accurate and the scheme defines a one-parameter family when $c$ is varied and adjusted for different goals $[43,44]$.

For illustration, published results are presented in Figure 6 to show the efficiency of the method. For comparison, red color marks the step $\beta$-function of four massless fermion flavors in the fundamental representation from an earlier publication [43]. The new published result for the step $\beta$-function of eight massless fermion flavors in the fundamental representation is marked with green color in the plot [45]. The step $\beta$-function of the sextet model of this report is marked with blue color in the plot from a recent publication [46]. This result ruled out an earlier claim of an infrared fixed point (IRFP) in the $\mathrm{g}^{2}=6$ range of the renormalized coupling which would have implied a conformal phase for the sextet model [47].

It is important to note that an IRFP was reported in the $\mathrm{g}^{2}=6.0-6.4$ range of the renormalized coupling with twelve massless fermions in the fundamental representation [48]. This IRFP is based on the study of the scale-dependent running coupling from the gradient flow with the choice $c=0.2$ and has been generally accepted as the most convincing evidence that the model with twelve massless flavors in the fundamental representation is inside the conformal window. Recent work 
using larger $c$ values in the gradient flow scheme does not find an IRFP in the reported range [49]. Further work would be required to resolve the tension between these two findings.

To establish the volume dependent running coupling without an IRFP in the range of renormalized couplings which can be reached within limitations of the method should be only one important part of the analysis. Equally important is to find independent and direct evidence for $\chi \mathrm{SB}$ to rule out the conformal phase. In this report we presented a preponderance of evidence for $\chi \mathrm{SB}$ in the sextet model. For added consistency, in the non-perturbative phase with $\chi \mathrm{SB}$ we are interested in a scale-dependent and volume independent renormalized coupling. At fixed lattice size, bare coupling, and fermion mass $\mathrm{m}$ we determine the appropriate flow time $\mathrm{t}\left(\mathrm{g}^{2}, \mathrm{~m}\right)$ to match any targeted flow-dependent renormalized coupling $g^{2}$ calculated from $\left\langle\mathrm{t}^{2} \mathrm{E}(\mathrm{t})\right\rangle$. Assuming that the footprint of the operator on the gradient flow is sufficiently small compared to the Compton wavelength of the pion for p-regime analysis, the dependence of $t\left(\mathrm{~g}^{2}, \mathrm{~m}\right)$ on $\mathrm{m}$ can be replaced by $\mathrm{t}\left(\mathrm{g}^{2}, \mathrm{M}_{\pi}^{2}\right)$ in $\chi \mathrm{PT}$ of pion dynamics with linear dependence of $\mathrm{t}\left(\mathrm{g}^{2}, \mathrm{M}_{\pi}^{2}\right)$ on $\mathrm{M}_{\pi}^{2}$ in leading order [50]. Any residual finite volume dependence can be corrected in $\chi \mathrm{PT}$. A scale dependent and volume independent step beta function can be determined from this procedure in the phase with $\chi \mathrm{SB}$. The analysis is far from complete in the sextet model. Plans for similar calculations in the fundamental rep with twelve flavors, motivated by our newest results, would be interesting to consider.

\section{Computational framework}

We developed promising and well-tested new methods to overcome some limitations of more conventional lattice BSM methods. They include the analysis of Goldstone dynamics with mixed actions, the chiral condensate, and spectroscopy in crossover from the p-regime to the $\varepsilon$-regime, close to the chiral limit of the theory. As a candidate theory of the composite Higgs particle the new approach requires new runs and a mixed valence action with excellent chiral properties of valence fermions. The required fermion mass range of the gauge configurations is below the range of our existing run set. Runs in the range from $\mathrm{m}=0.0010$ to $\mathrm{m}=0.0003$ operate in the crossover region from the p-regime to the $\varepsilon$-regime and runs with valence fermions in the $\mathrm{m}=0.0003-0.0001$ range reach further down in the fermion mass range of the RMT analysis. Three gauge couplings are needed to control the continuum limit.

Our physics analysis includes spectroscopy, the Dirac spectra, and the running coupling in capacity computing mode with new configuration generation plans. The disconnected diagrams of the low mass $0^{++}$state are extremely demanding on gpu resources. The conventional spectroscopy including analysis with a series of valence fermion masses in mixed actions, Dirac spectra including RMT, and configuration generation for the volume dependent running coupling also require large gpu resources for every given run parameter set in large volumes.

We use the tree-level Symanzik-improved gauge action for all simulations reported here. The conventional $\beta=6 / \mathrm{g}^{2}$ lattice gauge coupling is defined as the overall factor in front of the wellknown terms of the Symanzik lattice action. Its values are in the $\beta=3.20-3.30$ range of the proposed ensemble generation. The link variables in the staggered fermion matrix are exponentially smeared with two stout steps [25]; the precise definition of the staggered stout action was given in [26]. The RHMC algorithm is deployed in all runs. The fermion flavor doublet requires rooting in the algorithm. For molecular dynamics time evolution we apply multiple time scales [27] and the 
Omelyan integrator [28]. Our error analysis of hadron masses uses correlated fitting with double jackknife procedure on the covariance matrices. The time histories of the fermion condensate, the plaquette, the topological charge, and correlators are used to monitor autocorrelation times in the simulations.

The composite Higgs theory of this report requires a code structure which is distinct from lattice QCD in one important way. The fermions in QCD are in the fundamental representation of the SU(3) gauge group and represented in the code by a complex vector $\mathrm{q}_{\mathrm{i}}$, with color index $\mathrm{i}=1,2,3$. In the sextet model the fermions are represented as a two-index symmetric tensor $T_{i j}$ which is constructed as the symmetric part of the tensor $\mathrm{q}_{\mathrm{i}} \mathrm{q}_{\mathrm{j}}$.

The staggered Dirac operator, represented in matrix form and built from the SU(3) gauge link variables, has to be applied to the fermions field during the CG iteration steps consuming the dominant part of the ensemble generating evolution code. We developed two versions of the code. In the vector code, using the 6-dimensional vector representation of the fermions, the $\mathrm{SU}(3)$ gauge link matrices $U_{i j}$ are brought in from main memory and from the product $U_{i j} \cdot U_{k l}$ on each link a new 6x6 matrix $V_{m n}, m, n=1,2, \ldots 6$ is constructed which acts on the $Q_{n}$ vectors as a $V_{m n} \cdot Q_{n}$ matrixvector product. It is possible, however, to keep the Dirac matrix-vector algebra in the original two-index symmetric representation which entirely eliminates the overhead required to reconstruct the $6 \times 6$ matrix $V_{m n}$ and directly use the product of SU(3) link matrices on each link in tensor representation. The load on the memory bandwidth remains unchanged and on the BG/Q platform with excellent balance of the network bandwidth and memory bandwidth this leads to a factor of two reduction in flops and to a corresponding speedup factor in performance.

The code, with high utilization of the fast BG/Q network and with register level optimization of the local code on the nodes, was thoroughly tested for weak scaling and strong scaling across a large number of nodes on the BG/Q platform. The efficiency of the code in CG performance is 29 percent of the peak speed of the BG/Q platform on $48^{3} \times 96$ lattices on one rack with little loss of efficiency on half-rack. The numbers illustrate our efficient BG/Q code in lattice gauge theory applications of staggered fermions.

\section{Acknowledgement}

We acknowledge support by the DOE under grant DE-SC0009919, by the NSF under grants 0970137 and 1318220, by the DOE ALCC award for the BG/Q Mira platform of Argonne National Laboratory, by OTKA under the grant OTKA-NF-104034, and by the Deutsche Forschungsgemeinschaft grant SFB-TR 55. Computational resources were provided by the Argonne Leadership Computing Facility under an ALCC award, by USQCD at Fermilab, by the University of Wuppertal, by The Juelich Supercomputing Center on Juqueen and by the Institute for Theoretical Physics, Eotvos University. We are grateful to Szabolcs Borsanyi for his code development for the BG/Q platform. We are also grateful to Sandor Katz and Kalman Szabo for their code development for the CUDA platform [51]. KH wishes to thank the Institute for Theoretical Physics and the Albert Einstein Center for Fundamental Physics at the University of Bern for their support. 


\section{References}

[1] D. D. Dietrich, F. Sannino and K. Tuominen, Phys. Rev. D 72, 055001 (2005) [hep-ph/0505059].

[2] F. Sannino and K. Tuominen, Phys. Rev. D 71, 051901 (2005) [hep-ph/0405209].

[3] D. K. Hong, S. D. H. Hsu and F. Sannino, Phys. Lett. B 597, 89 (2004) [hep-ph/0406200].

[4] L. Susskind, Phys. Rev. D 20, 2619 (1979).

[5] S. Weinberg, Phys. Rev. D 19, 1277 (1979).

[6] W. J. Marciano, Phys. Rev. D 21, 2425 (1980).

[7] Z. Fodor, K. Holland, J. Kuti, D. Nogradi, C. Schroeder and C. H. Wong, Phys. Lett. B 718, 657 (2012).

[8] Z. Fodor, K. Holland, J. Kuti, D. Nogradi, C. Schroeder and C. H. Wong, PoS LATTICE 2012, 024 (2012).

[9] Z. Fodor, K. Holland, J. Kuti, D. Nogradi and C. H. Wong, PoS LATTICE 2013, 062 (2014) [arXiv:1401.2176 [hep-lat]].

[10] Z. Fodor, K. Holland, J. Kuti, S. Mondal, D. Nogradi and C. H. Wong, PoS LATTICE 2014, 244 (2015).

[11] Z. Fodor, K. Holland, J. Kuti, S. Mondal, D. Nogradi and C. H. Wong, arXiv:1601.03302 [hep-lat].

[12] K. Kainulainen, K. Tuominen and J. Virkajarvi, Phys. Rev. D 75, 085003 (2007) [hep-ph/0612247].

[13] R. Foadi, M. T. Frandsen, T. A. Ryttov and F. Sannino, Phys. Rev. D 76, 055005 (2007) [arXiv:0706.1696 [hep-ph]].

[14] M. Antola, M. Heikinheimo, F. Sannino and K. Tuominen, JHEP 1003, 050 (2010) [arXiv:0910.3681 [hep-ph]].

[15] K. Kainulainen, J. Virkajarvi and K. Tuominen, JCAP 1002, 029 (2010) [arXiv:0912.2295 [astro-ph.CO]].

[16] E. Witten, Phys. Lett. B 117, 324 (1982).

[17] S. L. Adler and W. A. Bardeen, Phys. Rev. 182, 1517 (1969).

[18] R. S. Chivukula and T. P. Walker, Nucl. Phys. B 329, 445 (1990).

[19] P. Langacker and G. Steigman, Phys. Rev. D 84, 065040 (2011) [arXiv:1107.3131 [hep-ph]].

[20] S. Borsanyi et al., Science 347, 1452 (2015) [arXiv:1406.4088 [hep-lat]].

[21] E. Aprile et al. [XENON100 Collaboration], Phys. Rev. Lett. 109, 181301 (2012) [arXiv:1207.5988 [astro-ph.CO]].

[22] D. S. Akerib et al. [LUX Collaboration], Phys. Rev. Lett. 112, 091303 (2014) [arXiv:1310.8214 [astro-ph.CO]].

[23] Z. Fodor, K. Holland, J. Kuti, D. Nogradi and C. H. Wong, PoS LATTICE 2013, 089 (2014) [arXiv:1402.6029 [hep-lat]].

[24] Z. Fodor, K. Holland, J. Kuti, S. Mondal, D. Nogradi and C. H. Wong, PoS LATTICE 2014, 270 (2015).

[25] C. Morningstar and M. J. Peardon, Phys. Rev. D 69, 054501 (2004). 
[26] Y. Aoki, Z. Fodor, S. D. Katz, K. K. Szabo, JHEP 0601, 089 (2006).

[27] C. Urbach, K. Jansen, A. Shindler, U. Wenger, Comput. Phys. Commun. 174, 87-98 (2006).

[28] T. Takaishi, P. de Forcrand, Phys. Rev. E73, 036706 (2006).

[29] W. J. Lee and S. R. Sharpe, Phys. Rev. D 60, 114503 (1999)

[30] Z. Fodor, K. Holland, J. Kuti, D. Nogradi, C. Schroeder, Phys. Lett. B 703, 348 (2011) [arXiv:1104.3124 [hep-lat].

[31] C. Aubin and C. Bernard, Phys. Rev. D 68, 034014 (2003)

[32] S. R. Sharpe and R. S. Van de Water, Phys. Rev. D 71, 114505 (2005) [hep-lat/0409018].

[33] M. Gell-Mann, R. J. Oakes and B. Renner, Phys. Rev. 175, 2195 (1968).

[34] T. Banks and A. Casher, Nucl. Phys. B 169, 103 (1980).

[35] Z. Fodor, K. Holland, J. Kuti, S. Mondal, D. Nogradi and C. H. Wong, in preparation.

[36] L. Giusti and M. Luscher, JHEP 0903, 013 (2009) [arXiv:0812.3638 [hep-lat]].

[37] M. E. Peskin and T. Takeuchi, Phys. Rev. D 46, 381 (1992).

[38] R. Foadi, M. T. Frandsen and F. Sannino, Phys. Rev. D 87, 095001 (2013) [arXiv:1211.1083 [hep-ph]].

[39] G. Cacciapaglia and F. Sannino, JHEP 1404, 111 (2014) [arXiv:1402.0233 [hep-ph]].

[40] S. Di Chiara, R. Foadi, K. Tuominen and S. Tahtinen, arXiv:1412.7835 [hep-ph].

[41] Z. Fodor, K. Holland, J. Kuti, D. Nogradi and C. H. Wong, JHEP 1211, 007 (2012) [arXiv:1208.1051 [hep-lat]].

[42] M. Luscher, JHEP 1008, 071 (2010) [arXiv:1006.4518 [hep-lat]].

[43] Z. Fodor, K. Holland, J. Kuti, S. Mondal, D. Nogradi and C. H. Wong, JHEP 1409, 018 (2014).

[44] Z. Fodor, K. Holland, J. Kuti, S. Mondal, D. Nogradi and C. H. Wong, arXiv:1410.8801 [hep-lat].

[45] Z. Fodor, K. Holland, J. Kuti, S. Mondal, D. Nogradi and C. H. Wong, JHEP 1506, 019 (2015).

[46] Z. Fodor, K. Holland, J. Kuti, S. Mondal, D. Nogradi and C. H. Wong, JHEP 1509, 039 (2015).

[47] A. Hasenfratz, Y. Liu and C. Y. H. Huang, arXiv:1507.08260 [hep-lat].

[48] A. Cheng, A. Hasenfratz, Y. Liu, G. Petropoulos and D. Schaich, JHEP 1405, 137 (2014).

[49] C.-J. D. Lin, K. Ogawa and A. Ramos, JHEP 1512, 103 (2015) doi:10.1007/JHEP12(2015)103 [arXiv:1510.05755 [hep-lat]].

[50] O. Bar and M. Golterman, Phys. Rev. D 89, no. 3, 034505 (2014) [Erratum-ibid. D 89, no. 9, 099905 (2014)].

[51] G. I. Egri, Z. Fodor, C. Hoelbling, S. D. Katz, D. Nogradi and K. K. Szabo, Comput. Phys. Commun. 177, 631 (2007) [hep-lat/0611022]. 


\section{Figures}
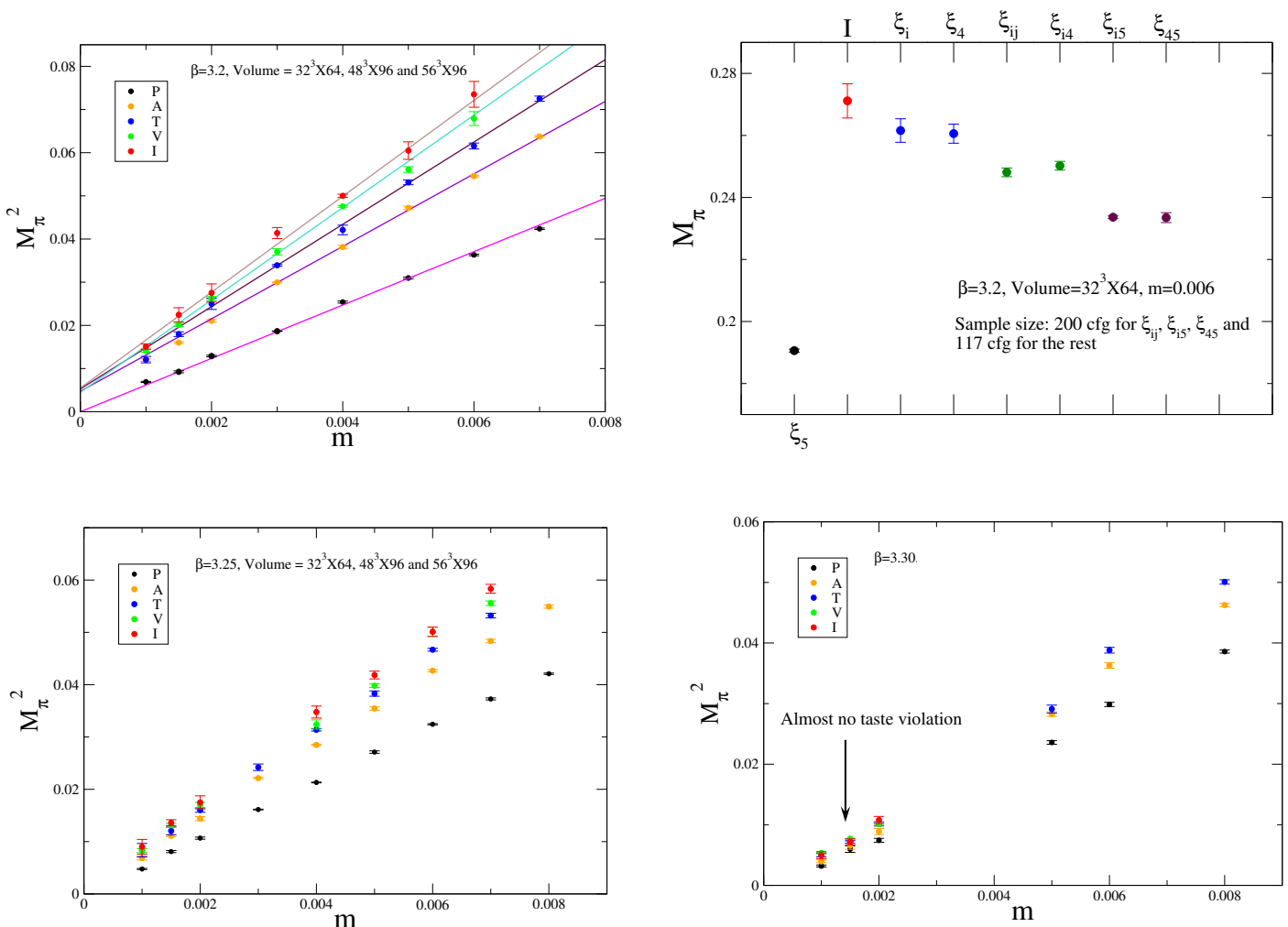

Figure 1: To illustrate cutoff dependent taste breaking effects, spectra of mass-deformed non-Goldstone pion states are shown from our newest data with the definition of the relevant correlators and quantum numbers given in [7,30]. In the fermion mass range of our data set the taste breaking pattern is different from QCD where the residual $\Delta$ mass shifts of the non-Goldstone pions are equispaced in the chiral limit with approximately degenerate $\mathrm{SO}(4)$ taste multiplets and with parallel slopes for finite fermion mass deformations of Goldstone and non-Goldstone pion states [29]. For example, as part of the equispaced split of degenerate $\mathrm{SO}(4)$ multiplets, the observed approximate split $\Delta_{\mathrm{ij}} \sim 2 \Delta_{\mathrm{sc}}$ of two multiplets in QCD appears to have collapsed in the sextet model. The other distinct difference from QCD is the non-parallel slopes which fan out in Goldstone and non-Goldstone mass deformations of the pion spectrum as shown. While the $\Delta$ additive mass shifts are LO taste breaking effects in the chiral Lagrangian [29,31], the taste breaking slope corrections $\delta$ can plausibly be identified with NLO analytic terms in the chiral analysis [32]. The corrected mass relation is $\mathrm{M}_{\mathrm{NLO}}^{2}=\mathrm{M}_{\mathrm{LO}}^{2}(1+\delta)$ where $\delta$ depends on the taste quantum number of the pion state. Several relations constrain the $\delta$ taste breaking corrections [32]. 

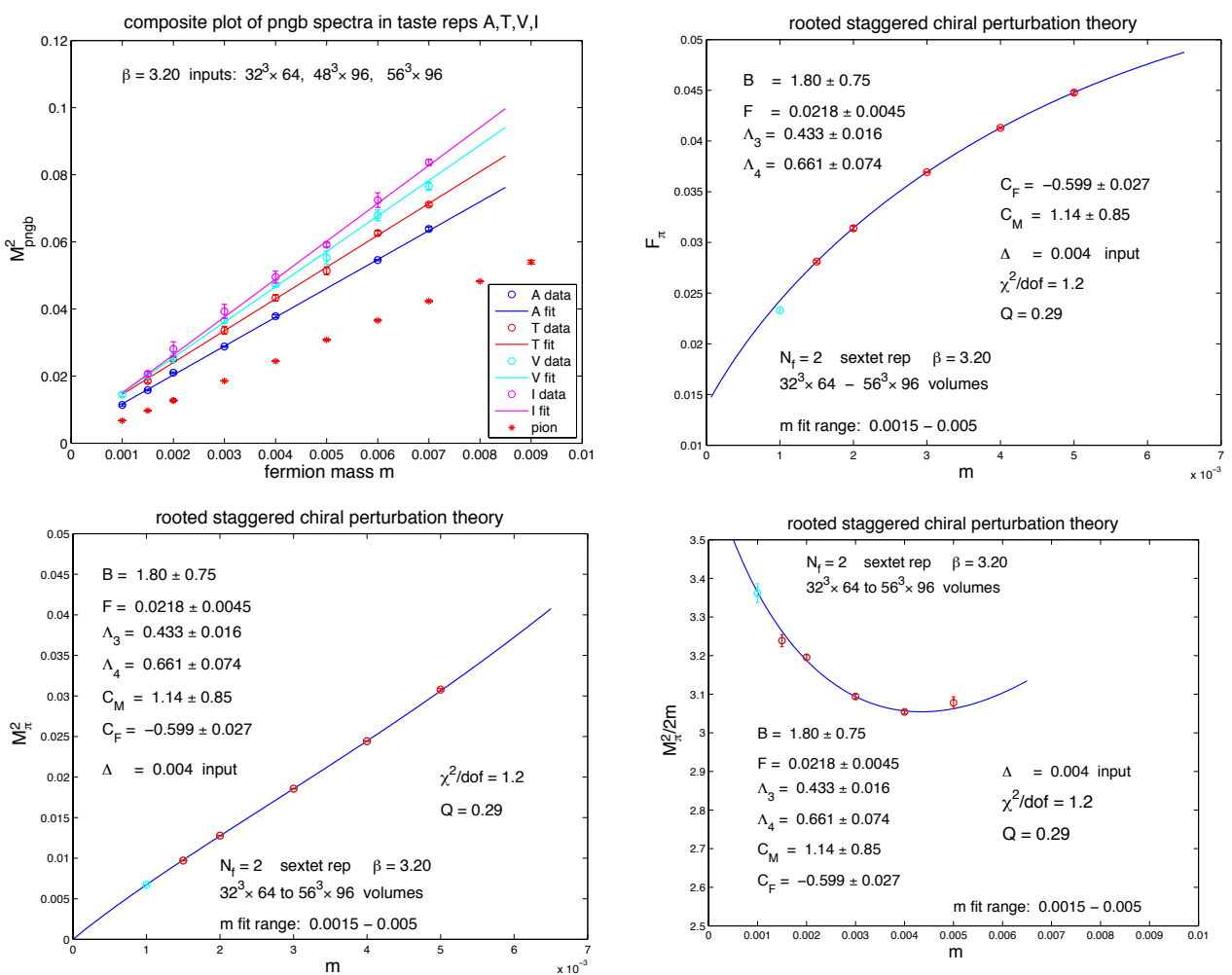

Figure 2: Preliminary results from rooted $\chi \mathrm{PT}$ are shown from fits at gauge coupling $\beta=3.20$ which corresponds to our coarser lattice of the two extended sets of gauge ensembles. The upper left panel shows the linear fits to the quadratic masses of the non-Goldstone pions to determine their mass shifts and slopes as input. The upper right panel shows the rooted $\chi \mathrm{PT}$ fit to $\mathrm{F}_{\pi}$ as a function of fermion mass deformations away from the chiral limit. The two lower panels show rooted $\chi \mathrm{PT}$ fits to $\mathrm{M}_{\pi}$ as a function of fermion mass deformations away from the chiral limit. We have similar analysis for $\mathrm{M}_{\pi}^{2}$ and $\mathrm{F}_{\pi}$ at $\beta=3.25$. 

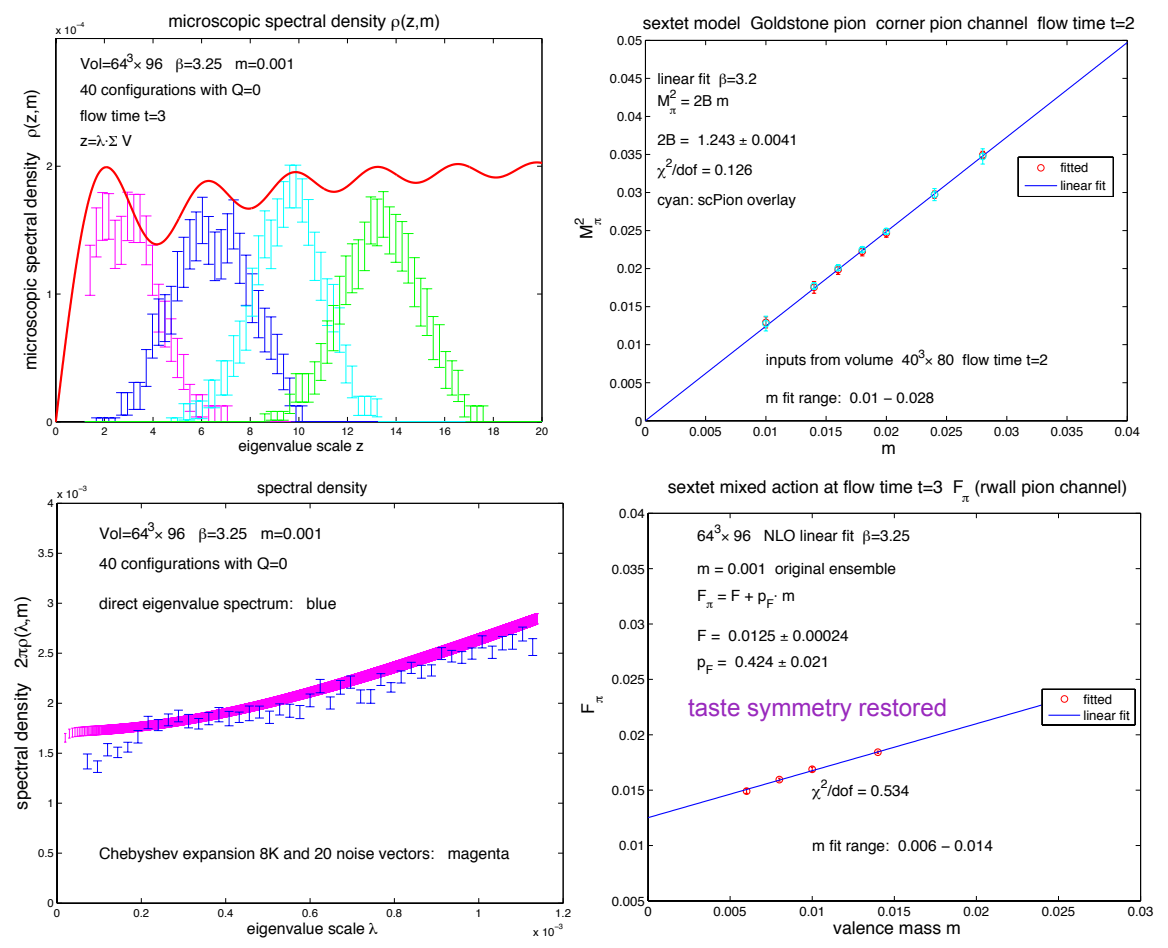

Figure 3: The newest test results are shown in the plot. The valence action is defined with a large number of small stout steps which corresponds to gradient flow time $t=3$ in cutoff units at each gauge coupling. We checked the eigenvalues of the Dirac operators which order into nearly degenerate quartets with the smeared gauge links of the gradient flow. The degenerate eigenvalues follow the index theorem count matching the topology of each gauge configuration as measured from the topological charge operator on the gradient flow. The upper left panel shows the RMT distribution of four degenerate quartets showing that the $\varepsilon$-regime is reached with the scaling variable $\lambda \Sigma_{\text {mixed }} \mathrm{V} \sim 10$ where the fermion mass is replaced by the scale of the gradient flow defined valence Dirac spectrum $(\mathrm{m} \rightarrow \lambda)$. The fermion condensate $\Sigma_{\text {mixed }}$, not RG invariant itself, is consistently determined from the gradient flow defined valence Dirac operator. The upper right panel illustrates the perfect degeneracy of the Goldstone pion with one selected non-Goldstone pion (scPion in the plot). We checked that the degeneracy holds for all non-Goldstone pion states. The lower left panel shows the infrared part of the directly calculated Dirac spectral density on the gauge configurations and its Chebyshev expansion based approximation. The lower right panel shows the pion decay constant $\mathrm{F}_{\pi}$ fitted with the mixed action for fixed sea mass as a function of valence masses. 

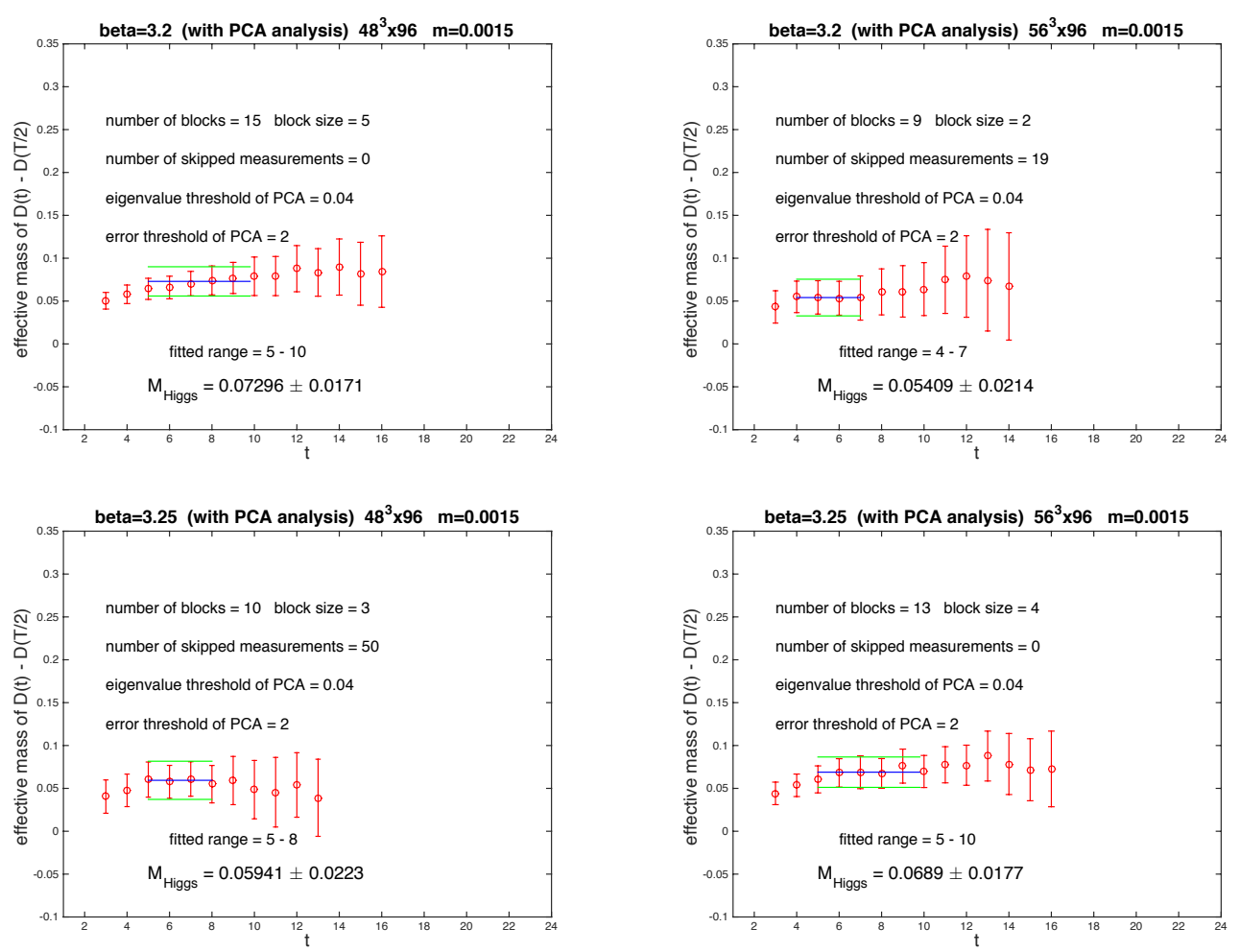

Figure 4: Representative fits of the low mass scalar from two ensembles using double Jackknife procedure on the covariance matrix with Principal Component Analysis (PCA). 


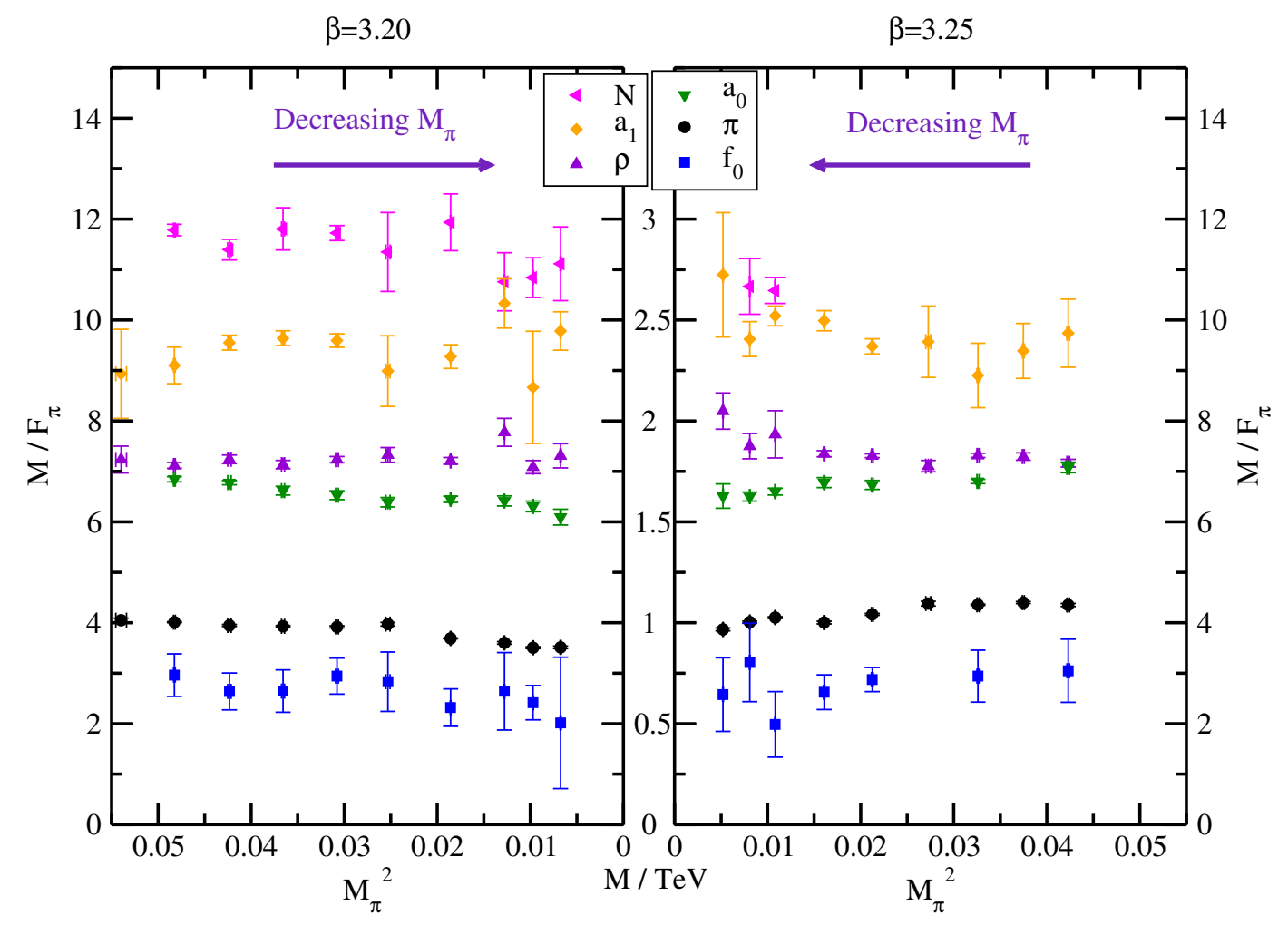

Figure 5: New resonance spectroscopy results are shown in the plot for illustration only. The scale is set by $\mathrm{F}=250$ $\mathrm{GeV}$ at both lattice spacings with caveats from discussions in Section 3 of the report. Any conclusion about $\chi \mathrm{SB}$ or conformal behavior from eyeballed inspection of the data would be inappropriate and misleading. 


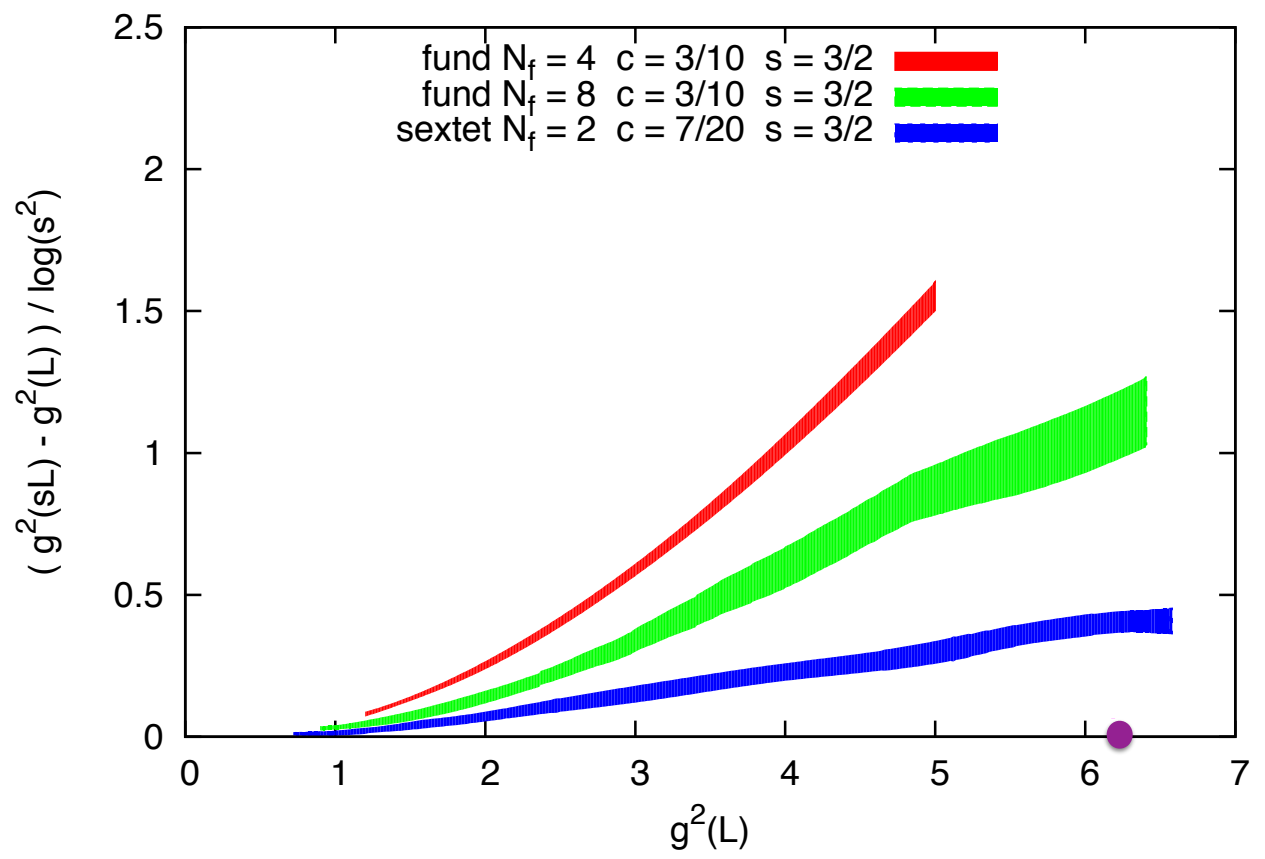

Figure 6: Comparative results are shown in the plot to illustrate the efficiency of the gradient flow based running coupling and $\beta$-function. For comparison, red color marks the step $\beta$-function of four massless fermion flavors in the fundamental representation from an earlier publication [43]. The new published result for the step $\beta$-function of eight massless fermion flavors in the fundamental representation is marked with green color in the plot [45]. The step $\beta$ function of the sextet model of this proposal is marked with blue color in the plot from a recent publication [46]. This result ruled out an earlier claim of an infrared fixed point (IRFP) in the $\mathrm{g}^{2}=6$ range of the renormalized coupling which would have implied conformal phase for the sextet model. The purple point in the plot marks the IRFP reported in the renormalized coupling with twelve massless fermions in the fundamental representation [48]. 